\title{
Efectos del agua de lastre sobre la calidad acuática de las zonas Portuarias de Callao, Pisco, Paita y Salaverry, Perú
}

\author{
Effects of ballast water on the aquatic quality of the Port areas of Callao, \\ Pisco, Paita and Salaverry, Peru
}

\author{
Rita Orozco 1, Alberto Lorenzo 1, Sonia Sánchez 1, Georgina Flores 1, Víctor Rebaza 1, Nancy Blas ${ }^{2}$ \\ Recibido: 01/07/2020 - Aprobado: 30/10/2020 - Publicado: 30/11/2020
}

\begin{abstract}
RESUMEN
El crecimiento de la industria naviera en el Perú y la ampliación de la zona portuaria del Callao, Pisco, Paita y Salaverry, permiten el ingreso de buques de gran calado. En los años 2009-2010, se reportó el ingreso de especies invasoras a través del agua de lastre en el Callao. Se realizó una evaluación del agua de lastre en los buques que arribaron al puerto del Callao en los años 2010 y 2012 .también se evaluó la calidad acuática de los puertos de Paita Salaverry, Callao y Pisco entre 2011 y 2013. Los resultados muestran que en el agua de lastre los recuentos de coliformes fueron bajos de < 2 a $8 \mathrm{NMP} / 100 \mathrm{ml}$, pero se registró a las bacterias Vibrio parahaemolyticus y V. vulnificus y especies fitoplanctónicas como Heterosigma akashivo y, Skeletonema costatum sp fueron las más representativas. Las aguas marinas de los puertos tenían valores normales de temperatura $<20$ ${ }^{\circ} \mathrm{C}$ y salinidades $>35.1$ ups, pero la calidad acuática fue afectada por actividades antropogénicas con valores elevados de coliformes termotolerantes en el Callao de 1,6x106 NMP/100ml. Las especies de fitoplancton dominantes producen floraciones algales Dictyocha fibula y Prorocentrum micans en Paita, Prorocentrum minimun, A. sanguineum en Salaverry en 2012, Heterosigma akashiwo y Akashiwo sanguinea,en el Callao en 2013. En el puerto de Pisco, las especies más abundantes fueron Cochlodinium polikrykoides en 2011 y Alexandrium peruvianum en mayo de 2012. El agua de lastre transporta especies exóticas invasoras que pueden causar graves efectos en el ecosistema marino, la salud humana y la economía, por lo cual debe fortalecerse el monitoreo de puertos de mayor tráfico marítimo.
\end{abstract}

Palabras clave: Agua de lastre; especies exóticas invasoras; Vibrio sp; fitoplancton; puertos.

\begin{abstract}
The growth of the shipping industry in Peru and the expansion of the port area of Callao, Pisco, Paita and Salaverry, allow the entry of large ships. In the years 2009-2010, the entry of invasive species through ballast water was reported in Callao. An assessment of the ballast water was carried out in the ships that arrived at the port of Callao in 2010 and 2012. The aquatic quality of the ports of Paita Salaverry, Callao and Pisco was also evaluated between 2011 and 2013. The results show that in ballast water coliform counts were low from <2 to 8 NMP / 100ml, the presence of Vibrio parahaemolyticus and V. vulnificus was recorded. The phytoplankton species such as Heterosigma akashivo and Skeletonema costatum $\mathrm{sp}$ were the most representative. The marine waters of the port areas had normal values of temperature $<20^{\circ} \mathrm{C}$ and salinities $>35.1$ ups, but the aquatic quality was affected by anthropogenic activities with high values of thermotolerant coliforms in Callao of $1.6 \times 10^{6} \mathrm{NMP} / 100 \mathrm{ml}$. Dominant phytoplankton species produce algal blooms Dictyocha fibula and Prorocentrum micans in Paita, Prorocentrum minimun, A. sanguineum in Salaverry in 2012, Heterosigma akashiwo and Akashiwo sanguinea, from Callao and in 2013a. In the port of Pisco, the most abundant species were Cochlodinium polikrykoides in 2011 and Alexandrium peruvianum in May 2012. Ballast water is transporting invasive alien species that can cause serious effects on the marine ecosystem, human health and the economy, for which should strengthen the monitoring of ports with greater maritime traffic.
\end{abstract}

Keywords: Ballast water; invasive alien species; Vibrio sp; phytoplankton; ports.

1 Instituto del Mar del Perú. Autor para correspondencia: ritaoro@imarpe.gob.pe

2 Dirección de Capitanías y Guardacostas Jr. Constitución 150-Callao. Esq. Gamarra y General Valle s/n Chucuito, Callao, Perú Apartado 22. 


\section{INTRODUCCIÓN}

El transporte marítimo es esencial para la economía mundial. Más del $90 \%$ del comercio mundial se realiza mediante buques, se ha estimado que cada año se desplazan por el mundo 10 millones de toneladas de agua de lastre, en las cuales y que pueden se transfieren más de 3000 especies de plantas y animales al día principalmente organismos planctónicos, necton y bacterias entre otros. Sin embargo, se conocen muchos casos de impacto negativo en el que especies del fitoplancton han sido transferidas por el agua de lastre y han sido causantes de mareas rojas, en los puertos de destino, ocasionando muertes masivas de peces o su intoxicación (Tamelander J., Riddering L., Haag F., 2010) e incluso la muerte de seres humanos En el panorama económico estas invasiones provocan daños a la pesca, los cultivos e incluso a la infraestructura portuaria ya que se adhieren organismos incrustantes, cuya eliminación es de alto costo; se calcula que en Estados Unidos el presupuesto puede alcanzar hasta cien mil millones de dólares anuales (Hurtado Gualán et al., 2017).

El Perú, tiene $3080 \mathrm{~km}$ de litoral costero, donde se distribuyen los terminales portuarios marítimos de Talara, Bayóvar, Punta Lobitos-Huarmey, Callao y Matarani En estos terminales, en los últimos años el transporte marítimo y la actividad portuaria han venido desarrollándose de forma acelerada, incrementando la infraestructura portuaria y logística naviera, logrando así, mayor tráfico de buques mercantes que llegan a nuestras costas.

La vigilancia de las actividades en los terminales portuarios cobra importancia al analizar el caso de Vibrio cholerae en el Perú. Esta bacteria es una especie invasora que tuvo un efecto sobre la salud fue el Vibrio cholerae, bacteria que produce el cólera el cual se dispersó rápidamente desde Chimbote llegando hasta Chile. En este contexto la OMI reconoció la descarga del cólera que afectó a cien mil personas en Perú en el año 1991, causando la muerte de más 5000 personas (Tamelander J., Riddering L., Haag F., 2010). Se estima que para la próxima década la invasión de especies exóticas y nocivas se triplicará debido al crecimiento del tráfico marítimo actual y por el aumento del número de buques. También podemos mencionar el transporte de muchas especies del fitoplancton a través del agua de lastre y que han ocasionado mareas rojas, causando muertes masivas de especies de peces o intoxicación e incluso la muerte de seres humanos (Tamelander J., Riddering L., Haag F., 2010). El agua de lastre contiene una diversidad de especies planctónicas y bentónicas en los tanques de lastre, siendo estos el principal vector de su transporte a través de barreras biogeográficas y que llegan a causar grandes cambios en la composición y la función de las comunidades ecológicas en agua dulce, estuarios y ecosistemas marinos (Williams et al., 1988; Carlton \& Geller, 1993).

La movilización de cargas transportadas por vía marítima es bastante diversa, pero las embarcaciones en general se caracterizan por movilizar importantes volúmenes de agua de mar como lastre para obtener la estabilidad necesaria para una navegación segura. En el Perú, según los datos de vigilancia de la Dirección de Capitanías y Guardacostas registrándose los mayores volúmenes en el Puerto de San Juan de Marcona en estos años (comm.pers N. Blas)

En el presente estudio se presentan los resultados la evaluación del agua de lastre del Puerto del Callao entre el 2010 al 2012 y la calidad acuática de los puertos de Pisco, Paita Y Salaverry entre el 2010 al 2013, también se presenta la composición de las especies de fitoplancton y bacterias.

\section{METODOS}

La evaluación del agua de lastre de los buques y de las áreas portuarias de Paita, Salaverry, Callao y Pisco (Figura 1), se efectuó entre los años 2010 al 2013.

\subsection{Muestreo del agua de lastre Puerto del Callao}

Se realizó el muestreo de agua de lastre entre el 2010 al 2012 en los barcos que arribaron al Puerto de Callao, en esta evaluación se obtuvo información del lugar de procedencia, los volúmenes totales de agua de lastre del tanque muestreado (Tabla 1).

El muestreo de los tanques de lastre de los buques se realizó de acuerdo a la guía para el muestreo de las aguas de lastre (G2) MEPC.173/58 (2008) conforme lo establece el Convenio del Agua de Lastre (Organización Marítima Internacional-OMI, 2004). A bordo se midió temperatura $\left({ }^{\circ} \mathrm{C}\right), \mathrm{pH}$ y se colecto muestras para determinar salinidad y nutrientes y análisis microbiológicos.

La salinidad se colectó en frascos de plástico de $250 \mathrm{ml}$ y las muestras para análisis microbiológicos y biológicos fueron pasadas a frascos de vidrio estériles y llevadas al Laboratorio de Microbiología Acuática del IMARPE para su posterior análisis. Para la colecta de las muestras de plancton se utilizó una red estándar de fitoplancton de 75 micras y malla abertura, las muestras fueron guardadas en frascos estériles de $250 \mathrm{ml}$.

\subsection{Muestras de agua $y$ plancton de puertos de Callao, Pisco, Paita y Salaverry 2011 al 2013}

Para la colecta de muestras de agua en los puertos seleccionados, se siguió las instrucciones establecidas en el Protocolo para el Monitoreo de Efluentes y Cuerpo marino Receptor, RM Nº03-2002-PE.

Para las determinaciones de parámetros físicos, químicos y microbiológicos se emplearon los siguientes métodos: Method 2540 D. APHA-AWWA-WEF, SM, 21st Ed., 2005: Determinación de Sólidos Suspendidos Totales.

Standard Methods for Examination of Water and Waste Water (APHA, 2005) 212T Edition: Cuantificación de bacterias del grupo coliformes siguiendo la metodología de tubos múltiples (Número Más Probable - NMP)

Standard International ISO $5815 \quad$ (1991): Determinación de la Demanda Bioquímica de Oxígeno

\section{$\underline{\text { Para los análisis de fitoplancton }}$}

Las muestras fueron preservadas con formalina neutralizada con bicarbonato de sodio, siguiendo las normas establecidas por Throndsen (1978). 


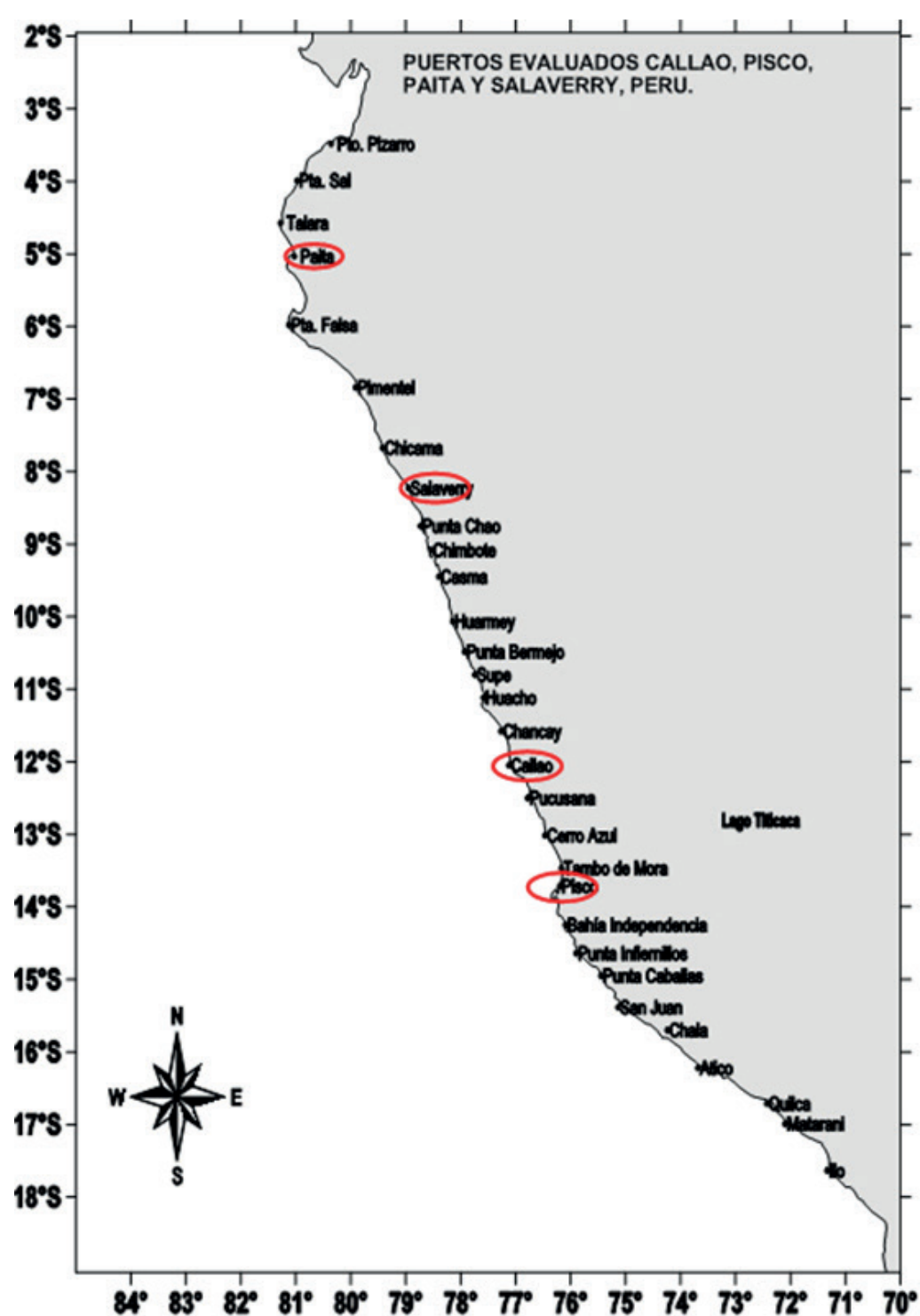

Figura 1. Puertos evaluados Callao, Pisco, Paita y Salaverry-Perú

Tabla 1. Relación de barcos muestreados en el Puerto del Callao 2010-2012

\begin{tabular}{lccccc}
\hline Tipo de nave & Procedencia & Fecha de muestreo & $\begin{array}{c}\text { Total agua lastre a } \\
\text { bordo }\left(\mathbf{m}^{3}\right)\end{array}$ & $\begin{array}{c}\mathbf{N}^{\circ} \text { de tanque }\left(\mathbf{m}^{3}\right) \\
\text { Vol. Agua de lastrel } \\
\text { tanque }\end{array}$ \\
\hline Porta Contenedor & Panama & $3 / 08 / 2010$ & $4,427.00$ & 4DBWB.TK(c) & no registra volumen \\
Ro - Ro & Manta, Ecuador & $24 / 08 / 2010$ & $4,113.00$ & WBT-1-CENTER & $580 \mathrm{m3}$ \\
Porta Contenedor & & $24 / 08 / 2010$ & $3,700.00$ & NO-28 WATERB.P & $253.5 \mathrm{m3}$ \\
Tanque petrolero quimiquero & Puerto llo, Peru & $6 / 09 / 2010$ & $2,500.00$ & 1.B.W(S) & $316 \mathrm{~m} 3$ \\
Carga General & Bahamas & $6 / 09 / 2010$ & 973.6 & FORE PEAK TANK & Tq. Vacío \\
Porta Contenedor & Antigua and Barbuda & $6 / 09 / 2010$ & $2,498.00$ & Tanque sellado & nd \\
Porta Contenedor & San Antonio, Chile & $20 / 12 / 2011$ & $2,498.00$ & WGTK4S & nd \\
Porta Contenedor & San Antonio, Chile & $20 / 12 / 2011$ & $4,177.00$ & WBS2FS P/S & 407 m3 \\
Porta Contenedor & Antofagasta, Chile & $14 / 02 / 2011$ & $3,358.00$ & 3A WTP & 300 m3 \\
Buk Carrier & Puerto Coronel, Chile & $14 / 02 / 2011$ & 271.72 & FP (Fore Peak) & nd \\
Buk Carrier & Panama & $12 / 06 / 2012$ & nd & 4WWB(P) & no registra volumen - \\
Porta Contenedor & Liberia & $12 / 06 / 2012$ & nd & nd & no registra volumen - \\
\hline
\end{tabular}

$\mathrm{Nd}=$ no dato 
El análisis cuantitativo se realizó con muestras de agua colectadas en cada uno de los tanques de agua de lastre. El recuento celular se realizó en base a la metodología de Utermöhl (1958), expresando los resultados en $\mathrm{N}^{\circ}$ cel $\mathrm{x}$ 103/L. Standard Methods for the Examination of Water and Wastewater 21st Edition, 2005 APHA-AWWA-WEF.

Determinación taxonómica de los diferentes organismos del fitoplancton Cupp (1943), Balech (1988), Sundström B.G. (1986).

\section{RESULTADOS}

\subsection{Agua de lastre de los buques}

3.1.1 Parámetros fisicoquímicos del agua de lastre entre el 2010-2012

En el Puerto del Callao se midieron los parámetros in situ como la temperatura del agua de lastre (Tabla 2), que varió de 16.0 a $22.8^{\circ} \mathrm{C}$, el $\mathrm{pH}$ entre 7.00 y 8.28. En el laboratorio se determinaron las concentraciones de oxígeno disuelto en el agua de lastre que variaron de 5.85 a $0.75 \mathrm{~mL} / \mathrm{L}$. el menor valor bajo de oxígeno que se encontró en el buque procedente del puerto de Antofagasta, Chile, es un medio hipóxico incompatible con la supervivencia de organismos vivos.

Las salinidades, presentaron valores menores a 34,8 ups en las naves de procedencia de Panamá, Guatemala y Colombia los que fueron diferentes a los patrones de Salinidad Superficial del Mar (SSM) (IMARPE, 2013) frente a Perú. En cambio, en las naves procedentes de los puertos Ilo, Perú, San Antonio y Antofagasta en Chile y el puerto de Liberia presentaron valores que estuvieron en el rango de 34,8 a 35,1 ups y fueron similares a las Aguas Costeras Frías de Perú según la descripción de las masas de agua frente a la costa peruana realizada por Zuta \& Guillén, (1970), (Tabla 3). Los resultados de nutrientes muestran valores muy elevados de fosfatos $(5,85 \mu \mathrm{M})$, en cambio los silicatos, presentan valores dentro de los rangos normales $(0-30 \mu \mathrm{M})$ para las aguas costeras de Perú. Respecto a los nitratos fueron menores $1 \mu \mathrm{M}$ lo que indicaría un gran consumo de este nutriente por los procesos de óxido-reducción con un aumento de los nitritos de 1,63 A $6,35 \mu \mathrm{M}$.

Tabla 2. Buques muestreados en el Callao entre el 2010 y 2013

\begin{tabular}{|c|c|c|c|c|c|}
\hline Puerto de origen & Tipo de Nave & Fecha & $\begin{array}{c}\text { Temperatura } \\
{ }^{\circ} \mathrm{C}\end{array}$ & $\mathrm{pH}$ & $\begin{array}{c}\text { Oxigeno Disuelto } \\
\mathrm{mL} / \mathrm{L}\end{array}$ \\
\hline Panama & Portacontendor & 3/08/2010 & 20.0 & 8.16 & 4.95 \\
\hline Guatemala & Ro-Ro & $24 / 08 / 2010$ & 16.2 & 8.15 & 3.00 \\
\hline Buenaventura, Colombia & Portacontendor & $24 / 08 / 2010$ & 18.3 & 8.28 & 5.35 \\
\hline Puerto llo & Tanque petrolero- quimiquero & $6 / 09 / 2010$ & 16.0 & 7.14 & 4.35 \\
\hline Bahamas & Carga general & $20 / 12 / 2010$ & 20.7 & 7.88 & 5.85 \\
\hline San Antonio, Chile & Portacontendor & $20 / 12 / 2010$ & 22.7 & 7.83 & 5.50 \\
\hline Antofagasta, Chile & Portacontendor & $14 / 02 / 2011$ & 16.5 & 7.00 & 0.75 \\
\hline Puerto Coronel & BulkCarrier & $14 / 02 / 2011$ & 20.6 & 7.14 & 5.85 \\
\hline Panama & BulkCarrier & $12 / 06 / 2012$ & 18.6 & 7.5 & 3.30 \\
\hline Liberia & Portacontendor & $13 / 06 / 2013$ & 18.6 & 7.5 & 4.57 \\
\hline
\end{tabular}

Tabla 3. Salinidad y nutrientes en el agua de lastre del Puerto del Callao (2010-2013)

\begin{tabular}{|c|c|c|c|c|c|c|c|}
\hline Puerto de origen & Tipo de Nave & Fecha & $\begin{array}{c}\text { Salinidad } \\
\%_{\circ}\end{array}$ & $\begin{array}{c}\text { Fosfatos } \\
\qquad \mu \mathrm{M} /\end{array}$ & $\begin{array}{c}\text { Silicatos } \\
\mu \mathrm{M}\end{array}$ & $\begin{array}{c}\text { Nitratos } \\
\mu \mathrm{M}\end{array}$ & $\begin{array}{c}\text { Nitritos } \\
\mu \mathrm{M}\end{array}$ \\
\hline Panama & Portacontendor & $3 / 08 / 2010$ & 33.3 & 0.25 & 12.51 & 2.73 & 0.35 \\
\hline Guatemala & Ro-Ro & $24 / 08 / 2010$ & 33.5 & 0.47 & 12.75 & 2.92 & 0.06 \\
\hline Buenaventura, Colombia & Portacontendor & $24 / 08 / 2010$ & 29.5 & 0.37 & 12.19 & 2.28 & 0.06 \\
\hline Puerto llo & Tanque petrolero- quimiquero & 6/09/2010 & 35.2 & 0.27 & 11.63 & 1.63 & 0.06 \\
\hline Bahamas & Carga general & $20 / 12 / 2010$ & 34.6 & 1.54 & 21.82 & 6.27 & 0.2 \\
\hline San Antonio, Chile & Portacontendor & $20 / 12 / 2010$ & 35.0 & 0.86 & 5.95 & 4.77 & 0.11 \\
\hline Antofagasta, Chile & Portacontendor & $14 / 02 / 2011$ & 35.0 & 1.47 & 0.4 & 6.35 & 0.9 \\
\hline Puerto Coronel & BulkCarrier & $14 / 02 / 2011$ & 35.1 & 5.82 & 18.4 & 3.63 & 3 \\
\hline Panama & BulkCarrier & $12 / 06 / 2012$ & 35.0 & 0.31 & 12.35 & 2.5 & 0.21 \\
\hline Liberia & Portacontendor & $13 / 06 / 2013$ & 35.0 & ND & ND & ND & ND \\
\hline
\end{tabular}

$\mathrm{ND}=$ No dato 


\subsubsection{Análisis microbiológicos del agua de lastre}

Los resultados de los análisis microbiológicos, los recuentos de E. coli variaron de $<2$ a 42 ufc/ $100 \mathrm{ml}$ (Tabla 4). En todas las muestras y los recuentos de bacterias heterótrofas viables variaron de $<30$ a 3.0 x 104 ufc/100ml. Se registró crecimiento de Vibrio sp. propias de los ecosistemas acuáticos y pueden encontrarse como células de vida libre o asociadas al plancton (Kaneko \& Colwell, 1975). Es así como varios estudios han demostrado que los vibrios son simbiontes con varios grupos de zooplancton (Louis et al., 2003), en el caso de copépodos ha sido reportado Vibrio cholerae sobre los sacos de huevos y sus piezas bucales (Huq et al., 1983). Existe una fuerte evidencia de que el agua de lastre de los buques de carga son el transporte importante de vectores de cólera y otras enfermedades relacionadas con el Vibrio según McCarthy \& Khambaty (1994), Mimura et al. (2005), Rivera et al. (2012) han atribuido a la descarga de agua de lastre la difusión de Vibrio cholerae $\mathrm{O} 1$ de las zonas portuarias a los ambientes costeros eutróficos, afectando pesquerías, cultivos, el turismo y la salud.

En las muestras de agua de lastre se realizó la investigación de Vibrio cholerae los cuales fueron aisladas y posteriormente identificadas mediante pruebas bioquímicas convencionales y el uso de los kits API $20 \mathrm{NE}$, dieron negativo para Vibrio cholerae. Pero se identificaron otras especies de Vibrio como Vibrio alginolyticus (54\%) que predominó en número seguido de Vibrio parahaemolyticus $(23 \%)$ es una bacteria halófila capaz de originar gastroenteritis en seres humanos y Vibrio vulnificus con $15 \%$, es una bacteria que encuentra un ambiente adecuado en las aguas marinas especialmente en zonas cálidas y también puede producir patologías en humanos (Figura 2).

3.1.3 Análisis de fitoplancton en el agua de Lastre en el Puerto de Callao 2010-2011

Los principales grupos de fitoplancton en agua de lastre en el Callao durante el 2010 y 2011 fueron las diatomeas,

Tabla 4. Resultados de análisis microbiológicos por puerto de origen y tipo de nave años 2010-2012

\begin{tabular}{|c|c|c|c|c|c|}
\hline Puerto de origen & Tipo de nave & Fecha & $\begin{array}{c}\text { E. Coli } \\
\text { (ufc/100 ml) }\end{array}$ & $\begin{array}{c}\text { Vibrio sp } \\
\text { (p/a) }\end{array}$ & $\begin{array}{l}\text { Heterótrofos } \\
\text { (ufc } / 100 \mathrm{ml} \text { ) }\end{array}$ \\
\hline Panamá & Portacontenedor & $3 / 08 / 2010$ & 0 & + & $<30$ \\
\hline Guatemala & Ro- Ro & $24 / 08 / 2010$ & 42 & + & $2.2 \times 10^{2}$ \\
\hline Buenaventura, Colombia & Portacontenedor & $24 / 08 / 2010$ & 16 & + & $2.1 \times 10^{3}$ \\
\hline Puerto Ilo, Peru & Tanque petróleo, quimiquero & 6/09/2010 & 0 & + & $3.0 \times 10^{4}$ \\
\hline Bahamas & Carga General & $20 / 12 / 2010$ & 33 & + & $5.4 \times 10^{2}$ \\
\hline San Antonio, Chile & Portacontenedor & $20 / 12 / 2010$ & 12 & + & $2.8 \times 10^{2}$ \\
\hline Antofagasta, Chile & Portacontenedor & $14 / 02 / 2011$ & 0 & + & $4.8 \times 10^{2}$ \\
\hline Puerto Coronel Chile & Bulk Carrier & $14 / 02 / 2011$ & 0 & + & $3.2 \times 10^{2}$ \\
\hline Panamá & Bulk Carrier & $12 / 06 / 2012$ & 8 & + & $3.0 \times 10^{2}$ \\
\hline Liberia & Portacontenedor & $13 / 06 / 2012$ & 2 & + & $4.3 \times 10^{2}$ \\
\hline
\end{tabular}

$\mathrm{P}=$ presencia $\mathrm{A}=$ Ausencia

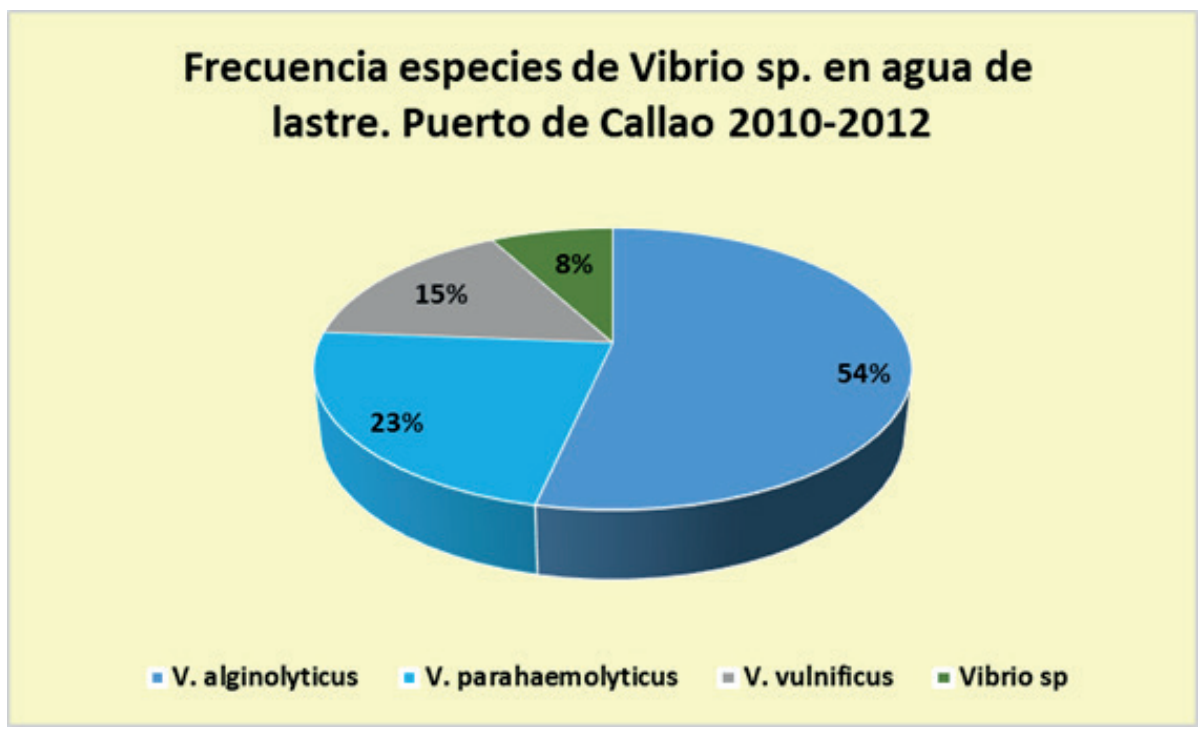

Figura 2. Frecuencia especies de Vibrio sp. en agua de lastre. Puerto de Callao 2010-2012 
dinoflagelados, microflagelados y otros grupos taxonómicos correspondiente al zooplancton como copépodos y larvas de bivalvos (Tabla5) Se ha observado relación de puerto de origen con la presencia y abundancia de las especies. En setiembre del 2010, en un barco procedente del Puerto de Ilo, en el agua de lastre se registró una temperatura de $16^{\circ} \mathrm{C}$ y 35.2 ups para relacionar con la presencia de las diatomeas y dinoflagelados, se presentó la especie Heterosigma akashiwo, que es un flagelado desnudo de pequeño tamaño, productor de Floraciones Algales Nocivas (FAN) que presentó valores de hasta $3,6 \times 10^{9}$ y Skeletonema costatum $2,49 \times 10^{4} \mathrm{cel} / \mathrm{L}$ diatomea con características oportunista y rangos amplios de distribución. También se registraron de un barco procedente de Colombia especies de ambientes oceánicos como Lioloma delicatulum, Bacteriastrum delicatulum, Coscinodiscus marginatus y C. curvatulus. En general la mayoría de las especies se encuentran registradas para Perú, según lo reportado por Ochoa, et al. 1999 a excepción de las especies de los géneros Navícula sp y Glenodinium $s p$.

\subsubsection{Análisis estadístico y comunitario de las especies} de fitoplancton

Se realizó la clasificación comunitaria de especies por buque y procedencia, utilizando el programa estadístico PAST 4.02 y se obtuvo un dendrograma de similitud Jaccard $\left(I_{J}\right)$ para calcular la similaridad de especies de fitoplancton entre los buques y su procedencia, observándose 3 agrupamientos de baja similitud, los valores de este índice relativamente más elevados lo presentaron los buques procedente de CSCLSao Paulo (Buenaventura Colombia) y Contielectra (Chile) respectivamente, presentando dominancia de especies en número de Nitzschia sp y Eutreptiella marina . El segundo agrupamiento lo presentaron los buques APL Managua y Mateo3 procedentes de Chile con dominancia de especies de Gynmodiales y Skeletonema costatum y el tercer agrupamiento con menor valor de similitud fueron los buques Cliper Makishio (puerto Ilo-Peru) y Vega Leader (Guatemala) presentando dominancia en número de especies Heterosigma akashivo y Chaetoceros lorenzianus( Figura 3)

También se calculó los índices de Diversidad entre los buques que arribaron al Puerto del Callao su procedencia y las especies registradas. Según el Índice de Shannon y Wienner (H') (Pla Laura, 2006) se encontró bajos valores de diversidad cuyo rango vario de 0.84 a 1.69 , esta condición se debe a que estas especies se encuentran sometidas a estrés por su permanencia de largos periodos de tiempo en el agua de lastre de los buques. Del total de especies encontradas dominaron en número los microflagelados y en términos de diversidad el buque SCLSao Paulo procedente de Buenaventura Colombia presento la mayor diversidad de Heterosigma akashivo.

La identificación del plancton lo realizo la Dra. Noemí Ochoa de la Universidad Nacional de San Marcos.

\subsection{Calidad acuática de las zonas portuarias de Salaverry, Callao y Pisco 2011 y 2013}

Las calidades acuáticas de zonas portuarias de Paita, Salaverry, Callao y Pisco fueron evaluadas a nivel superficial (Tabla 6). Los resultados muestran que la Temperatura superficial del mar (TSM) y la salinidad del período evaluado tienen las características descritas por Graco et al. (2007) en condiciones normales, en la zona norte en Paita presento valores propios de Aguas Subtropicales Superficiales (temperatura $<22^{\circ} \mathrm{C}$ y salinidades $>35.1$ ups. Las bahías de Salaverry, Callao y Pisco presentaron características propias de aguas costeras frías, con temperatura $<20^{\circ} \mathrm{C}$ y salinidades entre 34.8 y 35.1 ups.

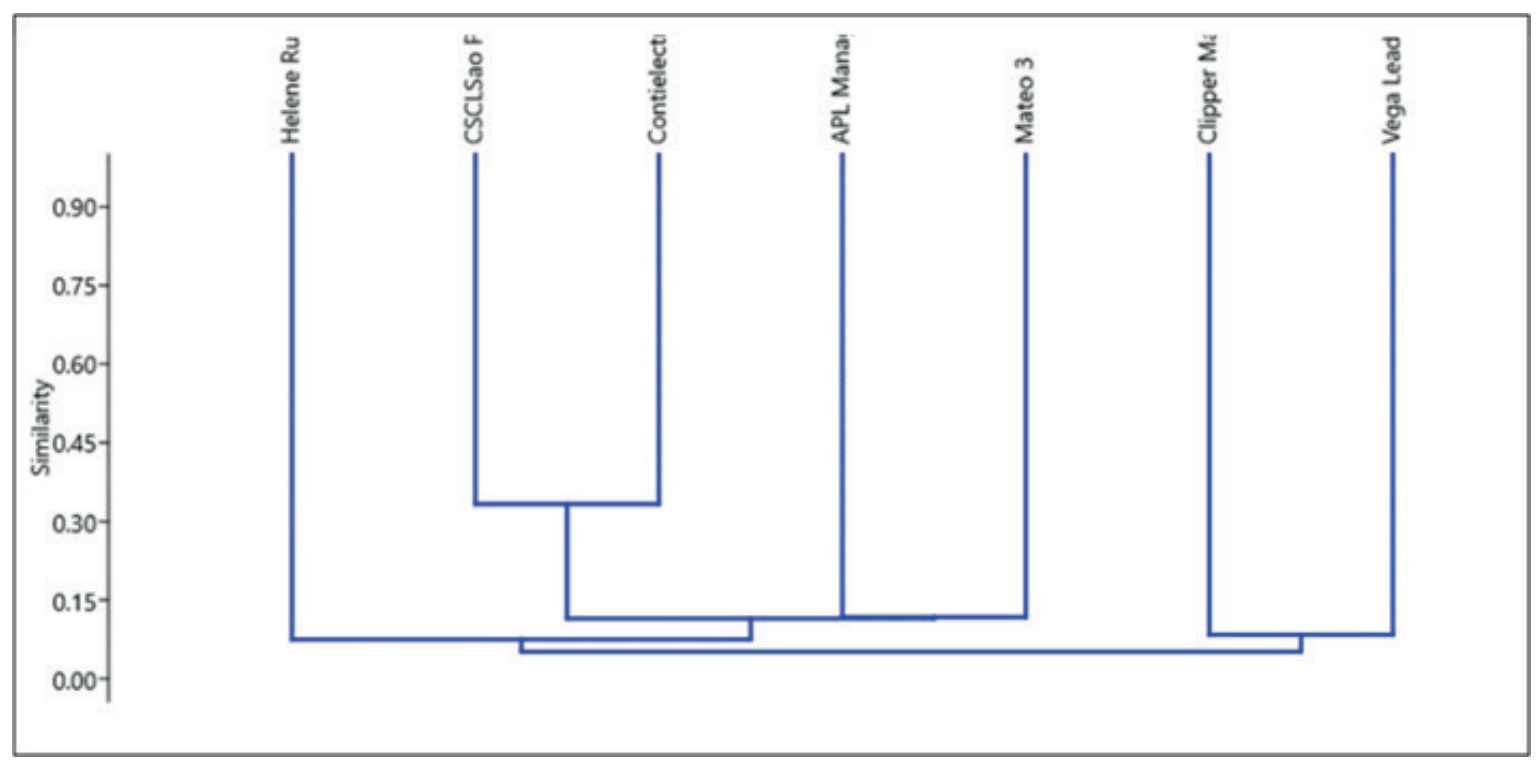

Figura 3. Dendrograma de similaridad de especies por buque y procedencia en el Puerto del Callao. 
Tabla 5. Análisis de fitoplancton de agua de lastre de los buques del Puerto de Callao 2010-2011

\begin{tabular}{|c|c|c|c|c|c|c|c|}
\hline Fecha & 24-Ago-10 & 24-Ago-10 & 6-Set-10 & 20-Dic-10 & 20-Dic-10 & 14-Feb-11 & 14-Feb-11 \\
\hline Buque & Vega Leader & CSCLSao Paulo & $\begin{array}{c}\text { Clipper } \\
\text { Makishlo }\end{array}$ & $\begin{array}{c}\text { APL } \\
\text { Managua }\end{array}$ & Contielectra & Mateo 3 & $\begin{array}{c}\text { Helene } \\
\text { Russ }\end{array}$ \\
\hline Procedencia & Guatemala & Buenaventura & Puerto llo & $\begin{array}{c}\text { San } \\
\text { Antonio } \\
\text { Chile }\end{array}$ & $\begin{array}{l}\text { San Antonio } \\
\text { Chile }\end{array}$ & $\begin{array}{l}\text { Antofagasta } \\
\text { Chile }\end{array}$ & $\begin{array}{c}\text { Puerto } \\
\text { Coronel, } \\
\text { Chile }\end{array}$ \\
\hline Actinocyclus octonarius & 40 & 0 & 40 & 0 & 2320 & 0 & 0 \\
\hline Amphora sp & 0 & 0 & 0 & 0 & 0 & 100 & 0 \\
\hline Amphripora sp & 0 & 0 & 0 & 0 & 0 & 180 & 0 \\
\hline Asterionellopsis glacialis & 0 & 83 & 0 & 0 & 0 & 0 & 0 \\
\hline Bacteriastrum delicatulum & 0 & 1041 & 0 & 0 & 0 & 0 & 0 \\
\hline Cerataulina pelagica & 0 & 0 & 0 & 0 & 0 & 0 & 20 \\
\hline Chaetoceros debilis & 160 & 0 & 0 & 0 & 0 & 0 & 0 \\
\hline Chaetoceros lorenzianus & 520 & 0 & 0 & 0 & 0 & 0 & 0 \\
\hline Chaetoceros sp & 0 & 42 & 0 & 0 & 0 & 0 & 0 \\
\hline Cocconeis sp & 0 & 0 & 0 & 0 & 0 & 1000 & 0 \\
\hline Coscinodiscus centralis & 0 & 333 & 0 & 0 & 680 & 0 & 20 \\
\hline Coscinodiscus curvatulus & 0 & 375 & 0 & 0 & 260 & 0 & 0 \\
\hline Coscinodiscus marginatus & 0 & 0 & 0 & 0 & 20 & 0 & 0 \\
\hline Coscinodiscus perforatus & 0 & 83 & 0 & 0 & 1120 & 0 & 0 \\
\hline Cylindrotheca closterium & 0 & 167 & 0 & 20 & 0 & 0 & 0 \\
\hline Entomoneis alata & 0 & 0 & 0 & 0 & 0 & 60 & 0 \\
\hline Grammatophora angulosa & 0 & 0 & 0 & 0 & 0 & 0 & 40 \\
\hline Gyrosigma sp & 0 & 0 & 0 & 0 & 0 & 0 & 20 \\
\hline Lauderia annulata & 0 & 0 & 0 & 220 & 0 & 0 & 0 \\
\hline Licmophora abbreviata & 0 & 0 & 80 & 0 & 0 & 0 & 0 \\
\hline Lioloma delicatulum & 0 & 83 & 0 & 0 & 0 & 0 & 0 \\
\hline Navicula sp. & 0 & 4166 & 0 & 0 & 2000 & 1500 & 0 \\
\hline Nitzschia sp & 0 & 4166 & 0 & 0 & 2500 & 40 & 0 \\
\hline Pleurosigma sp & 0 & 0 & 0 & 20 & 20 & 0 & 40 \\
\hline Rhizosolenia chuni & 0 & 0 & 0 & 500 & 0 & 0 & 0 \\
\hline Skeletonema costatum & 0 & 2083 & 0 & 280 & 0 & 24940 & 0 \\
\hline Thalassionema nitzschioides & 140 & 83 & 0 & 220 & 0 & 0 & 0 \\
\hline Thalassiosira angulata & 0 & 0 & 0 & 0 & 2500 & 0 & 0 \\
\hline Thalassiosira sp. & 0 & 0 & 0 & 0 & 0 & 40 & 0 \\
\hline TOTAL DE DIATOMEAS & 860 & 12705 & 120 & 1260 & 11420 & 27860 & 140 \\
\hline Dinophysis tripos & 0 & 42 & 0 & 0 & 0 & 0 & 0 \\
\hline Glenodinium sp & 0 & 0 & 0 & 0 & 0 & 5500 & 0 \\
\hline Gyrodinium sp & 0 & 0 & 0 & 0 & 0 & 500 & 0 \\
\hline Protoperidinium mendiolae (quistes) & 0 & 0 & 0 & 0 & 0 & 380 & 0 \\
\hline TOTALDE DINOFLAGELADOS & 0 & 42 & 0 & 0 & 0 & 6380 & 0 \\
\hline Eutreptiella marina & 0 & 125 & 140 & 0 & 15000 & 0 & 0 \\
\hline Gymnodiales & 0 & 0 & 155200000 & 4500 & 0 & 3000 & 0 \\
\hline Heterosigma akashivo & 0 & 0 & 3425600000 & 0 & 0 & 0 & 0 \\
\hline Microflagelados & 0 & 0 & 19200000 & 0 & 0 & 0 & 0 \\
\hline TOTAL DE MICROFLAGELADOS & 0 & 125 & 3600000140 & 4500 & 15000 & 3000 & 0 \\
\hline TOTAL DE FITOPLANCTON & 860 & 12872 & 3600000260 & 5760 & 26420 & 37240 & 140 \\
\hline Copepodos calanoides & 0 & 0 & 160 & 0 & 0 & 0 & 0 \\
\hline Estadio Nauplios & 0 & 0 & 60 & 0 & 0 & 0 & 0 \\
\hline Larvas de bivalvos & 0 & 0 & 20 & 0 & 0 & 0 & 0 \\
\hline
\end{tabular}


Tabla 6. Parámetros oceanográficos en los puertos de Paita, Salaverry, Callao y Pisco en los años 2011 y 2013

\begin{tabular}{lcccc}
\hline Bahías & $\begin{array}{c}\text { Temperatura } \\
{ }^{\circ} \mathrm{C}\end{array}$ & $\begin{array}{c}\text { Salinidad } \\
\text { psu }\end{array}$ & $\begin{array}{c}\text { Oxigeno } \\
\mathrm{mg} / \mathrm{L}\end{array}$ & $\mathrm{pH}$ \\
\hline Paita & $15.90-22.76$ & $35.103-35.337$ & $0.94-5.62$ & $7.29-8.00$ \\
Salaverry & $14.54-18.49$ & $34.836-35.099$ & $3.35-6.52$ & $7.43-7.80$ \\
Callao & $14.19-18.24$ & $34.836-35.100$ & $1.16-3.58$ & $6.71-8.53$ \\
Pisco & $15.57-22.01$ & $34.454-34.718$ & $0.00-14.66$ & $7.64-8.02$ \\
\hline
\end{tabular}

Fuente: Instituto del Mar del Perú

El oxígeno disuelto tuvo una fuerte variación entre los valores máximos y mínimos, registrándose valores de anoxia en el área de Pisco hasta periodos de máxima oxigenación posiblemente debido a una floración algal (0,0- $14.66 \mathrm{mg} / \mathrm{L})$, Callao y Paita presentaron valores que según la escala propuesta por (Tyson \& Pearson, 1991) tienen niveles de oxigenación: óxicos $(8,0$ a 2,0 mL.L1) subóxicos (desde 0,2 hasta 0,1 mL.L-1) anóxicos $(0,0$ mL.L-1. En contraste a ello en el área de Salaverry presento valores homogéneos que variaron de 3.35 a $6.52 \mathrm{~mL} / \mathrm{L}$. $\mathrm{El} \mathrm{pH}$ presento un rango de variación no muy notable a diferencia que en Salaverry

En la Tabla7, los sólidos suspendidos totales en el Callao presentaron valores que variaron de 114 a $146 \mathrm{mg} / \mathrm{L}$ en el periodo 2011 y 2013, pero menor a otras evaluaciones Paita y Salaverry presentaron sus máximas concentraciones de 150 a $256.39 \mathrm{mg} / \mathrm{L}$ respectivamente en el año 2012. En general, los sólidos suspendidos totales fueron elevados en todas las zonas evaluadas solo en el 2013 se registraron menores valores que se adecuaron a $\operatorname{los}$ ECA $(50 \mathrm{mg} / \mathrm{L})$ y el DBO5 presento los valores más elevados en Callao, que excedieron ampliamente los ECA que establece $10 \mathrm{mg} / \mathrm{L}$ y $1000 \mathrm{NMP} / 100 \mathrm{~mL}$ para coliformes termotolerantes para actividades marino-costeras.
Tabla 7. Parámetros de calidad acuática en los puertos de Paita, Salaverry, Callao y Pisco en los años 2011 y 2013

\begin{tabular}{lccc}
\hline Bahias & $\begin{array}{c}\text { SST } \\
\mathrm{mg} / \mathrm{L}\end{array}$ & $\begin{array}{c}\text { DBO5 } \\
\mathrm{mg} / \mathrm{L}\end{array}$ & $\begin{array}{c}\text { CTT } \\
\text { NMP/100ml }\end{array}$ \\
\hline Paita & $20.63-256.39$ & $1-9.5$ & $2-2.3 \times 103$ \\
Salaverry & $8.00-146.00$ & $1-2.78$ & $2-2.4 \times 103$ \\
Callao & $3.63-183.0$ & $1-105.15$ & $2-2.4 \times 106$ \\
Pisco & $6.90-39.52$ & $1-8.29$ & $2-5.0 \times 102$ \\
\hline
\end{tabular}

SST $=$ Solidos Suspendidos Totales, DBO5 $=$ Demanda Bioquímica de Oxígeno, $\mathrm{CTT}=$ Coliformes Termotolerantes

\subsection{Nutrientes en las zonas costeras}

Durante el año 2011 y 2013, los nutrientes a nivel superficie por áreas (Tabla 8) en Callao presentaron las mayores concentraciones de fosfatos $(4,35$ y $8,00 \mu \mathrm{M})$, mientras que en Pisco en los tres años consecutivos (2011 - 2013) las concentraciones máximas estuvieron entre 4,55 y $6,07 \mu \mathrm{M}$ sobrepasando los valores normales $(3,5 \mu \mathrm{M})$. En cambio, en Salaverry los valores de fosfatos fueron menores a $1 \mu \mathrm{M}$. Respecto a los silicatos estos en Callao para el 2013 alcanzaron valores máximos hasta $33,96 \mu \mathrm{M}$, mientras que en Pisco en los años 2011 a 2013 los valores fueron muy altos mayores a $40 \mu \mathrm{M}$. Los nitratos en las tres zonas Salaverry, Callao y Pisco fueron reducidos encontrándose concentraciones menores a $5 \mu \mathrm{M}$ a excepción de Pisco en el año 2011 en donde se encontró un valor de 23,22 $\mu \mathrm{M}$. En referencia a los nitritos presentaron valores dentro de los rangos normales $(0-12 \mu \mathrm{M})$ en las tres áreas y años.

\subsection{Las comunidades plantónicas de las zonas portuarias de Paita, Salaverry y Callao entre 2011 y 2013}

En el Programa de Seguimiento de floraciones algales en el mar peruano que realiza Instituto del Mar del Perú, se evaluó las comunidades fitoplanctónicas de las zonas

Tabla 8. Rangos de Nutrientes en los puertos de Salaverry, Callao, Pisco en los años 2011- 2013

\begin{tabular}{|c|c|c|c|c|c|}
\hline Zonas Portuarias & Años & $\begin{array}{c}\text { Fosfatos } \\
\qquad \mu \mathrm{M}\end{array}$ & $\begin{array}{c}\text { Silicatos } \\
\qquad \mu \mathrm{M}\end{array}$ & $\begin{array}{l}\text { Nitratos } \\
\qquad \mu \mathrm{M}\end{array}$ & $\begin{array}{l}\text { Nitritos } \\
\qquad \mu \mathrm{M}\end{array}$ \\
\hline \multirow[t]{2}{*}{ Salaverry } & 2011 & $0,01-0,02$ & $0,46-1,11$ & $0,07-0,14$ & $0,02-0,07$ \\
\hline & 2012 & $0,12-0,56$ & $0,32-1,32$ & $0,00-0,04$ & $0,02-0,07$ \\
\hline \multirow[t]{3}{*}{ Callao } & 2011 & $3,18-8,00$ & $11,99-27,61$ & $1,82-5,84$ & $0,61-1,04$ \\
\hline & 2012 & $1,91-3,16$ & $13,43-21,30$ & $0,99-3,07$ & $0,18-0,99$ \\
\hline & 2013 & $1,84-4,35$ & $17,64-33,96$ & $0,17-2,02$ & $0,14-0,28$ \\
\hline \multirow[t]{3}{*}{ Pisco } & 2011 & $0,61-4,55$ & $2,40-48,87$ & $0,00-23,22$ & $0,00-1,92$ \\
\hline & 2012 & $1,32-6,76$ & $8,83-47,79$ & $0,07-4,55$ & $0,02-3,41$ \\
\hline & 2013 & $1,10-6,07$ & $16,69-43,78$ & $0,26-1,31$ & $0,02-1,96$ \\
\hline
\end{tabular}

Fuente: Instituto del Mar del Perú 
portuarias con el mayor tráfico de carga por operación: Paita, Salaverry y Callao entre 2011 y 2013.

En Paita en febrero del 2013, se observó al dinoflagelado atecado Gyrodinium sp., produjo una floración algal de tonalidad marrón rojizo intenso y alcanzó una concentración celular de $17,060 \mathrm{cel} / \mathrm{L}$, a Dictyocha fibula con 12,667 cel/L, seguida del dinoflagelado Prorocentrum micans $4067 \mathrm{cel} / \mathrm{L}$, también estuvieron presentes Gyrodinium sp., Ceratium dens yC. fusus v. seta.

En Salaverry en noviembre del 2012, se registró valores homogéneos de temperatura de 15,5 a $15,8^{\circ} \mathrm{C}$, la Salinidad de 34,813 a 35,333 ups, la comunidad fitoplanctónica estuvo conformada por diatomeas Thalassionema nitzschioides, Thalassiosira rotula, Coscinodiscus curvatulus, C. granii, C. centralis, y Lithodesmiun undulatum,. También cabe indicar la ocurrencia y abundancia de dinoflagelados cosmopolitas Ceratium furca y Protoperidinium depresum.

En el Puerto del Callao, en enero del 2011 asociados a temperatura superficial del mar, menores a $14,8{ }^{\circ} \mathrm{C}$ se observó las especies Eutreptiella gymnastica y Messodinium rubrum, y está última característica de los ambientes de afloramiento. Entre noviembre y diciembre del 2012 en un ambiente cálido a ligeramente cálido asociados a TSM de $18,1^{\circ} \mathrm{C}$ y $19,1^{\circ} \mathrm{C}$ respectivamente y con amplia presencia de aguas costeras frías, se reportaron especies nocivas como los dinoflagelados Dinophysis acuminata, D. caudata, D. rotundata y Prorocentrum minimum,. En el 2013, bajo condiciones ambientales de TSM que fluctuó entre $22.5^{\circ} \mathrm{C}$ y $15.3^{\circ} \mathrm{C}$, la salinidad alrededor de 35 ups y valores de oxígeno de $4.16 \mathrm{ml} / 1$ a $10.40 \mathrm{ml} / 1$, floraciones algales de las especies Heterosigma akashiwo, durante los meses de Marzo, Abril, Mayo y Julio; Akashiwo sanguinea, durante Mayo y Junio; y e Prorocentrum micans en el mes de Setiembre

En Pisco En bahía de Paracas/Pisco, destacó la presencia Cochlodinium polikrykoides en el verano del 2011 y Alexandrium peruvianum en el otoño 2012. En el verano del 2013 se registró un total de 7 especies potencialmente tóxica, en La Pampa (Bahía Independencia) destacó el Grupo Pseudonitzschia delicatissima especies que alcanzaron una máxima concentración de 2,200 cel/L. Otras especies que se presentaron fueron Alexandrium sp., Dinophysis acuminata, D. caudata, Prorocentrum minimum y Protoperidinium crassipes con densidades $(<900 \mathrm{cel} / \mathrm{L})$ que no representaron perjudiciales para la salud pública.

\section{CONCLUSIONES}

En el agua de lastre del Puerto del Callao se registró especies de fitoplancton predominando Heterosigma akashivo especie que representa un riesgo para el desarrollo la maricultura y recreación porque produce toxinas y también puede afectar la salud humana. Todas las especies se encuentran registradas para Perú, excepto las especies Navícula sp y Glenodinium sp, también se registraron especies de microflagelados no identificados con abundancia en número, y presencia de copépodos calanoides, estadios Nauplios y larvas de bivalvos potencialmente invasores.

En el agua de lastre se registraron bacterias como Vibrio parahaemolyticus y Vibrio vulnificus que afectan a la maricultura y a los peces e invertebrados que son destinados a consumo humano.

La información obtenida en este trabajo indica que alteración de los parámetros fisicoquímicos e incumplimiento de las directrices del Convenio de Agua de lastre, por los bajos de salinidad y que indican que no han realizado el recambio del agua de lastre a las 12 millas, también los recuentos elevados de bacterias y de fitoplancton.

Los puertos evaluados presentan contaminación y el Puerto del Callao es el más impactado por los elevados valores de coliformes termotolerantes máximas concentraciones de $1,6 \times 10^{6} \mathrm{NMP} / 100 \mathrm{ml}$. El monitoreo de las áreas portuarias de Paita, Salaverry Callao y Pisco permite conocer la calidad acuática, así como el registro de especies endémicas, y especies invasoras en estas áreas.

\section{AGRADECIMIENTOS}

Este trabajo se realizó en el marco Proyecto Regional Globallast GEF/UNDP/IMO- CPPS por DICAPI e IMARPE., agradecemos a los biólogos del Laboratorio de Fitoplancton y Producción Primaria y Laboratorio de Microbiología Acuática. En especial a la Dra. Noemi Ochoa $\dagger$ de la Universidad Nacional de San Marcos por su apoyo en la identificación por los organismos fitoplanctónicos.

\section{REFERENCIAS}

APHA. (2005). Standard Methods for the Examination of Water and Wastewater. AMERICAN PUBLIC HEALTH ASSOCIATION; AMERICAN WATER WORKS ASSOCIATION; WATER ENVIRONMENT FEDERATION. Standard Methods for the Examination of Water and Wastewater. https://doi.org/10.1016/00431354(82)90249-4

Balech, E. (1988). Los dinoflagelados del Atlántico sudoccidental. Publ. Espec. Inst. Esp. Oceanogr., 1, 310. http://www. repositorio.ieo.es/e-ieo/handle/10508/993

Carlton, J. T., \& Geller, J. B. (1993). Ecological Roulette: The global transport of nonindigenous marine organisms. Science, 261(5117), 78-82. https://doi.org/10.1126/ science. 261.5117 .78

Cupp, E. E. (1943). Marine Plankton Diatoms of the west Coast of North America. Bulletin of the Scripps Institution of Oceanography, 5(1), 199-207. https://escholarship.org/uc/ item/922945w8

Graco, M. I., Ledesma, J., Flores, G., \& Girón, M. (2007). Nutrientes, oxígeno y procesos biogeoquímicos en el sistema de surgencias de la corriente de Humboldt frente a Perú. In Revista Peruana de Biologia (Vol. 14, Issue 1, pp. 117-128). https://doi.org/10.15381/rpb.v14i1.2165 
Huq, A., Small, E. B., West, P. A., Huq, M. I., Rahman, R., \& Colwell, R. R. (1983). Ecological relationships between Vibrio cholerae and planktonic crustacean copepods. Applied and Environmental Microbiology, 45(1), 275-283. https://doi.org/10.1128/aem.45.1.275-283.1983

Hurtado Gualán, M., Hurtado Domínguez, M., Dahik Garzozi, Á., Flores Yagual, G., \& Garcia Aguilera, J. (2017). Estrategia Nacional para la Gestión de Agua de Lastre en Ecuador. Ecosistemas Marinos y Costeros, 59-392.

IMARPE- Instituto del Mar del Perú. (2013). Temperatura superficial del mar y anomalías térmicas, Salinidad Superficial del Mar. http://www.imarpe.pe/imarpe/index. php?id seccion $=\mathrm{I} 0178030103000000000000$

Kaneko, T., \& Colwell, R. R. (1975). Adsorption of Vibrio parahaemolyticus onto Chitin and Copepods. Applied Microbiology, 29(2), 269-274. https://doi.org/10.1128/ aem.29.2.269-274.1975

Louis, V. R., Russek-Cohen, E., Choopun, N., Rivera, I. N. G., Gangle, B., Jiang, S. C., Rubin, A., Patz, J. A., Huq, A., \& Colwell, R. R. (2003). Predictability of vibrio cholerae in chesapeake bay. Applied and Environmental Microbiology, 69(5), 2773-2785. https://doi.org/10.1128/AEM.69.5.27732785.2003

McCarthy, S. A., \& Khambaty, F. M. (1994). International dissemination of epidemic Vibrio cholerae by cargo ship ballast and other nonpotable waters. Applied and Environmental Microbiology, 60(7), 2597-2601. https://doi. org/10.1128/aem.60.7.2597-2601.1994

Mimura, H., Katakura, R., \& Ishida, H. (2005). Changes of microbial populations in a ship's ballast water and sediments on a voyage from Japan to Qatar. Marine Pollution Bulletin, 50(7), 751-757. https://doi.org/10.1016/j. marpolbul.2005.02.006

Organización Marítima Internacional-OMI. (2004). CONVENIO INTERNACIONAL PARA EL CONTROL Y LA GESTIÓN DEL AGUA DE LASTRE Y LOS SEDIMENTOS DE LOS BUQUES, 2004. Adopción: 13 de Febrero de 2004; Entrada En Vigor: 8 de Septiembre de 2017. http://www. imo.org/es/About/Conventions/ListOfConventions/ Paginas/International-Convention-for-the-Control-andManagement-of-Ships\%27-Ballast-Water-and-Sediments(BWM).aspx
Pla Laura. (2006). Biodiversidad: inferencia basada en el índice de shannon y la riqueza. In Interciencia (Vol. 31, Issue 8). http://ve.scielo.org/scielo.php?script=sci arttext\&pid=S0378-18442006000800008

Rivera, I. N. G., Souza, K. M. C., Souza, C. P., \& Lopes, R. M. (2012). Free-living and plankton-associated vibrios: Assessment in ballast water, Harbor areas, and coastal ecosystems in Brazil. Frontiers in Microbiology, 3(JAN). https://doi.org/10.3389/fmicb.2012.00443

Sundström B.G. (1986). The Marine diatom genus Rhizosolenia. A new approach to the taxonomy. Doctoral Thesis. Lund. https://www.algaebase.org/search/species/ detail $/ ? \mathrm{tc}=$ accept $\&$ species id $=59746$

Tamelander J., Riddering L., Haag F., M. J. (2010). Guidelines for Development of National Ballast Water Management Strategies. GloBallast Monographs, 18. www.imo.org

Throndsen, J. (1978). Preservation and storage. UNESCO Digital Library. https://unesdoc.unesco.org/ark:/48223/pf 0000030791? posInSet=1\&queryId=2810a6a7-4789-4f98b3ba-e 9 b5ef5c7c40

Tyson, R. V., \& Pearson, T. H. (1991). Modern and ancient continental shelf anoxia: An overview. Geological Society Special Publication, 58, 1-24. https://doi.org/10.1144/GSL. SP.1991.058.01.01

Utermöhl, H. (1958). Zur Vervollkomrnnung ver quantitativen Phytoplankton-Methodic. Mitt. Int. Verein. https://www. tandfonline.com/doi/abs/10.1080/05384680.1958.1190409 1

Williams, R. J., Griffiths, F. B., Van der Wal, E. J., \& Kelly, J. (1988). Cargo vessel ballast water as a vector for the transport of non-indigenous marine species. Estuarine, Coastal and Shelf Science, 26(4), 409-420. https://doi. org/10.1016/0272-7714(88)90021-2

Zuta, S., \& Guillén, O. (1970). Oceanografía de las aguas costeras del perú. In Boletin Inst. Mar. Perú-Callao (Vol. 2, Issue 5, pp. 157-324). http://biblioimarpe.imarpe.gob.pe/ handle/123456789/949 